\title{
Operations research confronting the crisis
}

\author{
Nikolaos Matsatsinis $^{1}$ - Yannis Siskos ${ }^{2} \cdot$ John Psarras $^{3}$
}

Published online: 12 November 2016

C Springer Science+Business Media New York 2016

In the highly volatile socio-economic environment that emerged due to the recent global crisis, new opportunities have arisen for the development and application of operations research (OR) methodologies. These challenges/opportunities involve a wide spectrum of areas that extend well beyond the economic and financial aspects of the crisis, as both public policy makers and corporate managers adjust their goals to the new context, where issues such as sustainable development, employment growth, social welfare, business ethics, and corporate governance, have gained much importance.

This special issue (SI) presents recent advances of OR models in areas where decision making has been affected by the crisis. On the methodological side, the SI presents new realistic and powerful OR models with direct practical contributions. These include new decision making approaches, formulations and solution algorithms, procedures for improving computational performance, as well as implementations for decision support systems. Case studies and papers presenting innovative applications in different application areas are also presented.

All submitted papers were reviewed by at least two referees in order to meet the standards of the Annals of Operations Research. The referees made every effort possible to ensure that the manuscripts accepted for publication reached a high standard. After the review process, a collection of 22 papers were selected for this special issue. The contents are grouped into the following five main thematic areas.

\footnotetext{
$\bowtie$ Nikolaos Matsatsinis

nikos@ergasya.tuc.gr

Yannis Siskos

ysiskos@unipi.gr

John Psarras

john@epu.ntua.gr

1 School of Production Engineering and Management, Technical University of Crete, Chania, Greece

2 Department of Informatics, University of Piraeus, Piraeus, Greece

3 School of Electrical and Computing Engineering, National Technical University of Athens, Athens, Greece
} 


\section{Management and finance}

In the paper of Xidonas, Doukas, Mavrotas, and Pechak, the need to develop alternative models able to address the current economic situation through the exploitation of sustainable patterns is studied. The innovation of this current study is the incorporation of Energy and Environmental Corporate Responsibility (EECR) in decision making, supporting particularly the development of a new model for investment evaluation. A bi-objective programming model is introduced in order to provide the Pareto optimal portfolios (Pareto set) based on the Net Present Value (NPV) of projects and the EECR score of firms.

Cadenillas and Huamán-Aguilar develop a stochastic debt control model to study the optimal government debt ceiling, or equivalently the optimal ceiling for government debt. They consider a government that wants to control its debt by imposing an upper bound or ceiling on its debt-to-GDP ratio.

Bovi and Cerqueti examine the way economic turmoils influence the lay agents' predictions of macroeconomic fundamentals, and for this they develop a dynamic stochastic optimization problem, which is solved by performing extensive Monte Carlo simulations.

Oliveira, Coelho, and Antunes study the interactions between the economy (at national, global, and local levels), the energy sector, and the corresponding impacts on the environment that inherently involve multiple axes of evaluation of distinct policies (economy-energyenvironment-social-E3S). They use input-output (IO) analysis for developing multiobjective models for assessing the trade-offs associated with those policies.

Benediktsdóttir, Bjarnadóttir, and Hansen present a methodology based on linear algebra and networks that creates groups of related parties based on ownership information, financial dependencies, business associations, and family ties. They apply the methodology retrospectively to highlight the large exposure violations and systemic risk leading up to the 2008 banking crises in Iceland.

Cont and Minca presents a network model for investigating the impact on systemic risk of central clearing of over the counter (OTC) credit default swaps (CDS). He proves that when interest rate swaps are cleared, central clearing of credit default swaps through a wellcapitalized CCP can reduce the probability and the magnitude of a systemic illiquidity spiral by reducing the length of the chains of critical receivables within the financial network.

Bilbao-Terol, Arenas-Parra, Cañal-Fernández, and Bilbao-Terol tackle two emerging streams in the financial literature: the behavioral portfolio theory with mental accounting and the socially responsible investment (SRI). They use goal programming formulations in order to reconcile the two choice frames and a fuzzy inference system to determine the amount of money allocated to each mental account.

In the paper of Damel, Le Thi, and Peltre, a way to model liquidity risk and the credit risk in best practices is proposed. The authors propose a heuristic approach to combine the numerous liquidity risk indicators with a logistic regression, and a methodology using stochastic diffusion for the interest rate because currently the yield curves are not liquid.

\section{Marketing}

Kipenis and Askounis propose a methodological framework to assess the main dimensions of an e-Participation system from the users' perspective by measuring users' satisfaction and highlighting its advantages. They also identify its shortcomings in order to propose areas for improvement. 
Tsafarakis considers the optimal product line design which is an NP-hard optimization problem in marketing that involves a number of decisions, such as product line length and configuration. The presented approach outperforms genetic algorithms and classic simulated annealing, through the use of crossover as a neighborhood operator, along with the restricted tournament selection as the replacement strategy of the evolutionary algorithm's population. The applicability of the proposed approach is illustrated through the case of the 2008 automotive industry crisis.

Carayannis and Grigoroudis consider innovation-driven competitiveness and propose a methodological measurement framework based on multiobjective mathematical programming models in order to study the linkage among national innovation, productivity, and competitiveness and discover potential performance patterns. The model is applied on a set of European countries for the period of 1998-2008.

In their paper, Gosse and Hurson consider the relationship between employee satisfaction and specific job characteristics. They view employees as internal customers of an organization, and their satisfaction as an important driver for business success, and assess job satisfaction of recent recruits in a large French company.

Tsafarakis, Saridakis, Matsatsinis, and Baltas introduce Differential Evolution (DE) to assist retailers in modifying their product portfolios in periods of economic recession and to facilitate strategic product assortment planning (PAP) decisions, related to (a) optimal variety of PL product categories, (b) optimal service level of private labels (PL) merchandise within a product category, and hence (c) optimal balance between PLs and National Brands (NBs) in a retailer's product portfolio. The proposed approach is illustrated through an implementation to an empirical dataset derived from a random sample of 1928 consumers who participated in a large scale computer assisted telephone survey during the recent economic crisis period.

\section{Logistics}

Duhamel, Santos, Brasil, Châtelet, and Birregah propose a mathematical model and heuristics for solving a multiperiod location-allocation problem in post-disaster operations, which takes into account the impact of distribution over the population. Logistics restrictions such as human and financial resources are considered.

Faghih-Roohi, Ong, Asian, and Zhang present a dynamic model of conditional value-atrisk (CVaR) measure for risk assessment and mitigation of hazardous material transportation in supply chain networks. The well-established market risk measure, CVaR, which is commonly used by financial institutions for portfolio optimizations, is investigated. A solution algorithm is finally proposed for solving the model, with verifications made using numerical examples and sensitivity analysis.

$\mathrm{Wu}, \mathrm{Xu}$, and $\mathrm{Xu}$ propose a multiple attribute group decision making (MAGDM) framework to facilitate lean practices performance evaluations. The paper deals with the consensus process and selection process for MAGDM problems based on the 2-tuple linguistic computation model. Finally, the developed MAGDM framework is applied to a lean practices evaluation problem for a commercial tobacco company's logistics distribution centers in China.

Yang, Yuan, Du, and Liang develop a decision support system based on the data envelopment analysis method for the construction of reserve network for China Red Cross based on three factors, including cost, risk, and utility. Four warehouses are selected and the optimal allocation strategies are obtained under the principles of equity and effectiveness that should be obeyed in humanitarian assistance. 


\section{Health}

In the paper of Fragkiadakis, Doumpos, Zopounidis, and Germain, a non-parametric modeling approach is employed to assess and analyze the efficiency of 87 Greek public hospitals over the period 2005-2009, using data envelopment analysis. The operational and economic aspects of the hospitals' operations are considered on the basis of their service/case mix and cost structure.

Mitropoulos, Kounetas, and Mitropoulos evaluate health centers in Greece, and identify factors impeding efficiency, with the aim of determining how their efficiency could be improved. Two alternative conceptual models are used to ensure the consistency of the efficiency results: one model focuses on production efficiency and the other on economic efficiency. Subsequently a second stage analysis is performed to account for the impact of explanatory variables on efficiency.

\section{Methodologies}

Artikis proposes a stochastic model and a conceptual framework of cindynics which is used for investigating the evolution of a complex system going through a crisis generated by the occurrence of a major risk.

Ahmed considers three crossover and ten mutation operators for genetic algorithms and then compares them for the quadratic assignment problem (QAB) on some benchmark QAPLIB instances. The experimental study shows the effectiveness of the sequential constructive crossover and the adaptive mutation operators for the problem.

Mahapatra, Mahapatra, and Roy introduce a new solution technique for a fuzzy non-linear programming problem with both decision variables and restriction being fuzzy in nature. The proposed procedure is applied to a complex system reliability model to evaluate the system reliability in a fuzzy environment. Finally, a numerical example is presented to illustrate the proposed fuzzy system.

We would like to express our sincerest thanks to all contributing authors for their highquality papers. We are also grateful to all reviewers who devoted considerable time and effort to carefully read the submissions in order to provide critical evaluation and constructive comments. 